\title{
Visualization of the Multichannel Seismocardiogram
}

\author{
Kim Munck ${ }^{1}$, Johannes J Struijk ${ }^{1}$, Kasper Sørensen ${ }^{1}$, Samuel E Schmidt ${ }^{1}$ \\ ${ }^{1}$ Aalborg University, Aalborg, Denmark
}

\begin{abstract}
The multichannel seismocardiography (mchSCG) project aims to develop the technology and knowledge-base to understand the distribution of vibration waves on the chest wall caused by cardio-mechanic events. This study focuses on the developing visualization methods for the vibration waves based on the multi-dimensional map obtained with the mchSCG equipment. We investigated four visualization methods. Vibration signals were collected in a four by four grid with 16 three-axis accelerometers placed on the chest of 11 healthy males. The visualization methods were investigated for their abilities to show temporal, spatial, and directional information. Of the four methods the SCG chart shows best temporal and small amplitude sensitivity information. Color plots provides an improved spatial overview. Tracking maps provide good directionality. The seismic mesh method is good at showing spatial and directionality information. Dependent on which signal aspects are of interest, the four visualization methods have their specific suited purposes. These visualization methods can assist further investigation of the vibration waves behavior and its relation to cardio-mechanic events.
\end{abstract}

\section{Introduction}

Seismocardiography (SCG) is a method for measurement of chest wall vibrations related to cardiac events [1]. Commonly, SCG involves measuring with an accelerometer on a single site on sternum, typically above the Xiphoid Process. However, recent studies have demonstrated that additional measurement sites reveal complementary information related to cardio-mechanic events [2].

Studies have proposed different techniques for multisite vibration maps. These techniques involve microphones [3], Laser Doppler Vibrometer [4], ultrasound waves [5], and accelerometers [6]. A common goal of these techniques is to quantify cardio-mechanic events in a simple and reproducible way.

This study aims to compare four approaches for visualization of multi-dimensional surface vibration for presentation and interpretation of multichannel SCG (mchSCG).

\section{Methods}

The four proposed visualization methods were based on existing methods and named the following in this study: SCG charts, color plots, tracking maps, and seismic mesh. The strength and weakness of the methods is described based on visualization abilities of the 11 subject's data.

Chest surface vibrations were recorded using mchSCG equipment, described in detail by Munck [7]. The mchSCG consist of 16 three-axis accelerometers placed on the subject's chest. The accelerometers were placed in a four by four grid with the accelerometer in location 3,2 placed above the Xiphoid Process, as illustrated by Fig. 1. Accelerometers were spaced $40 \mathrm{~mm}$ apart.

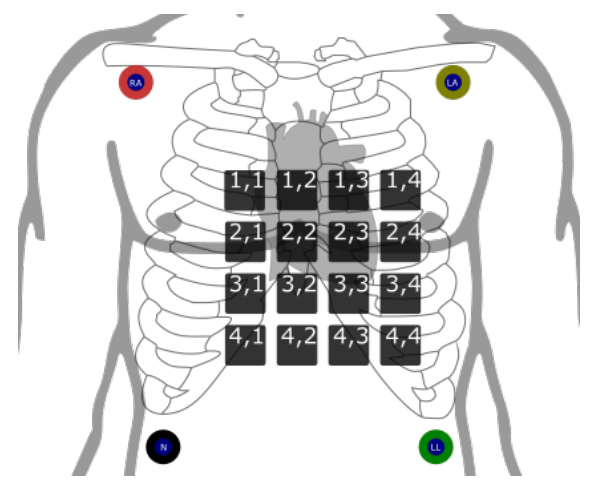

Figure 1. Accelerometer locations and ECG electrode positions. Accelerometer 3,2 is placed above the Xiphoid Process. Vibrations are defined in the positive directions of the three cardinal axes: frontal (left), vertical (superior), and sagittal (anterior).

ECG was measured synchronous as a reference signal. Signals were acquired for 5 minutes with $500 \mathrm{~Hz}$. Single segments of either one or three second length where used for the visualization methods. The SCG signals were filtered with a forwards-backwards low and high-pass Butterworth filter of $90 \mathrm{~Hz}$ and $0.05 \mathrm{~Hz}$ [8].

Recordings from 11 male subjects were used for the investigation of the visualization methods. The study was approved by the North Denmark Region Committee on Health Research Ethics: N-20170008. 


\subsection{SCG charts}

The SCG chart was inspired by the standard 12-lead $4 \times 3$ ECG chart, where three leads are shown at a time in four columns, with a rhythm strip at the bottom. There are one SCG trace for each accelerometer, and the signals is drawn in consistence with the accelerometers' grid locations. Three examples of the SCG chart are illustrated by Fig. 2, 3 , and 4 . One example for each of the three cardinal axes.

\subsection{Color plots}

Color plots are commonly used to show surface activities in the literature, such as Body Surface Potential Maps (BSPM). Since this visualization method does not include time, multiple snapshots or a video segment is necessary. Color plot use a four-dimensional spline interpolation increasing the spatial resolution. The fourdimensions was time, frontal location, vertical location, and the three-axis acceleration. A colored frame around each snapshot is used to represent time relative to the ECG and SCG. An example of the color plot is illustrated by Fig. 5, for one of the three cardinal axes.

\subsection{Tracking maps}

The tracking map visualization method are based on Schmidt et al., 2017 method to track the displacement of a single three-axis accelerometer [9]. Here the three-axis acceleration trajectory changed over time is plotted as a single line for each accelerometer. The color of the trajectory changed in correspondence with time. The colorbar below the ECG and SCG plot indicated the corresponding time. Black square represented the accelerometers location, whereas the vector from their location and to a given point on the trajectory was the acceleration at that given time. An example of the tracking map are illustrated in Fig. 6, where the visualization method can be viewed at two different angles.

\subsection{Seismic mesh}

The seismic mesh are based on how seismic surface waves is illustrated in the literature [10]. This method favored spatial information and use interpolated lines between the accelerometers to show the wavelengths. The interpolation increased the grid resolution from 4 by 4 sites to 25 by 25 sites. The interpolation is illustrated as solid lines between existing accelerometers and dotted lines between the added sites. This visualization method does not include time and multiple snapshots or video segment are necessary to represent time. Black squares represented existing accelerometer locations. Additionally, an average collective vibration of all the accelerometers was shown as a circle with a plus, to show the collective motion of the torso. As an example of the seismic mesh a single frame from a video segment was illustrated by Fig. 7, presented from two different viewing angles.

\section{Results}

\subsection{SCG chart}

The SCG chart is useful when investigating temporal similarities and signal magnitude differences, due to the grid and signals alignment. The between beat variation can also be observed. However, the scaling had to be adaptive, because the SCG signal has a larger amplitude variation between subject then compared to ECG signals

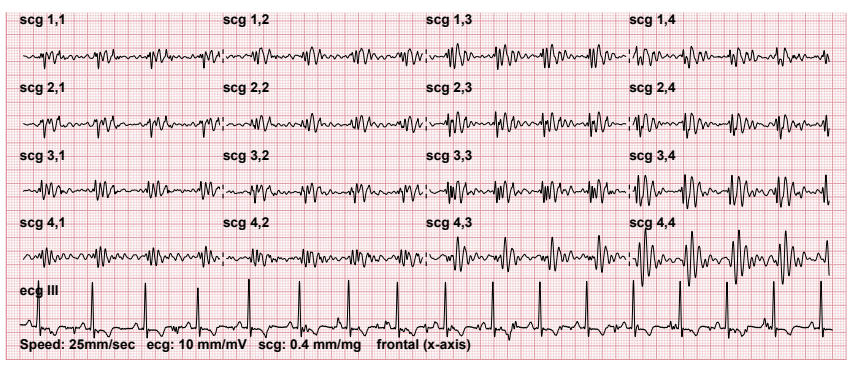

Figure 1 Example of SCG chart for frontal accelerations. Each larger grid square is 5 by $5 \mathrm{~mm}$, which scales to $200 \mathrm{msec}$ and $12.5 \mathrm{mg}$. Data in figure is from subject 7.

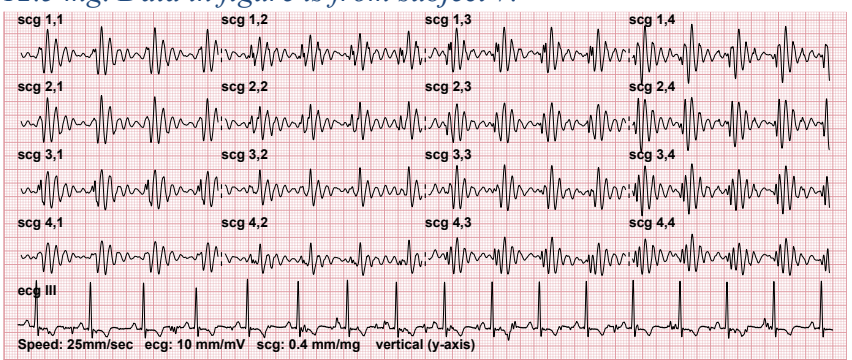

Figure 2 Example of SCG chart for vertical accelerations. Each larger grid square is 5 by $5 \mathrm{~mm}$, which scales to $200 \mathrm{msec}$ and $12.5 \mathrm{mg}$. Data in figure is from subject 7.

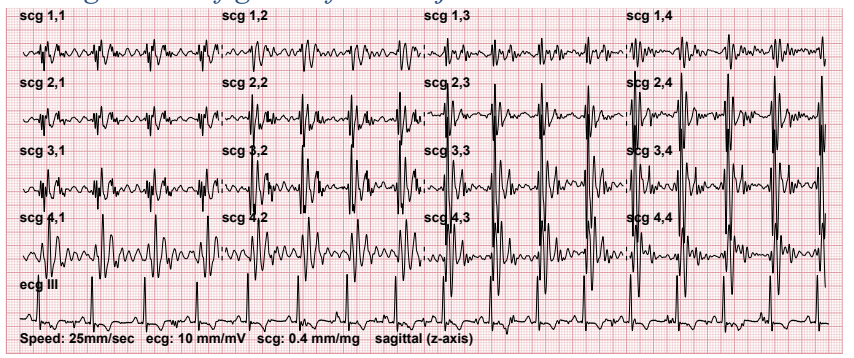

Figure 3 Example of SCG chart for sagittal accelerations. Each larger grid square is 5 by $5 \mathrm{~mm}$, which scales to $200 \mathrm{msec}$ and $12.5 \mathrm{mg}$. Data in figure is from subject 7. 


\subsection{Color plot}

Since the method used interpolation the of vibration acceleration waves can be investigated a higher spatial resolution. Interpolation also makes it possible to find a more precise spatial location, timing and amplitude of some fiducial points. Similar to the SCG chart only one axis is shown in one color plot, making it difficult to compare axial information.

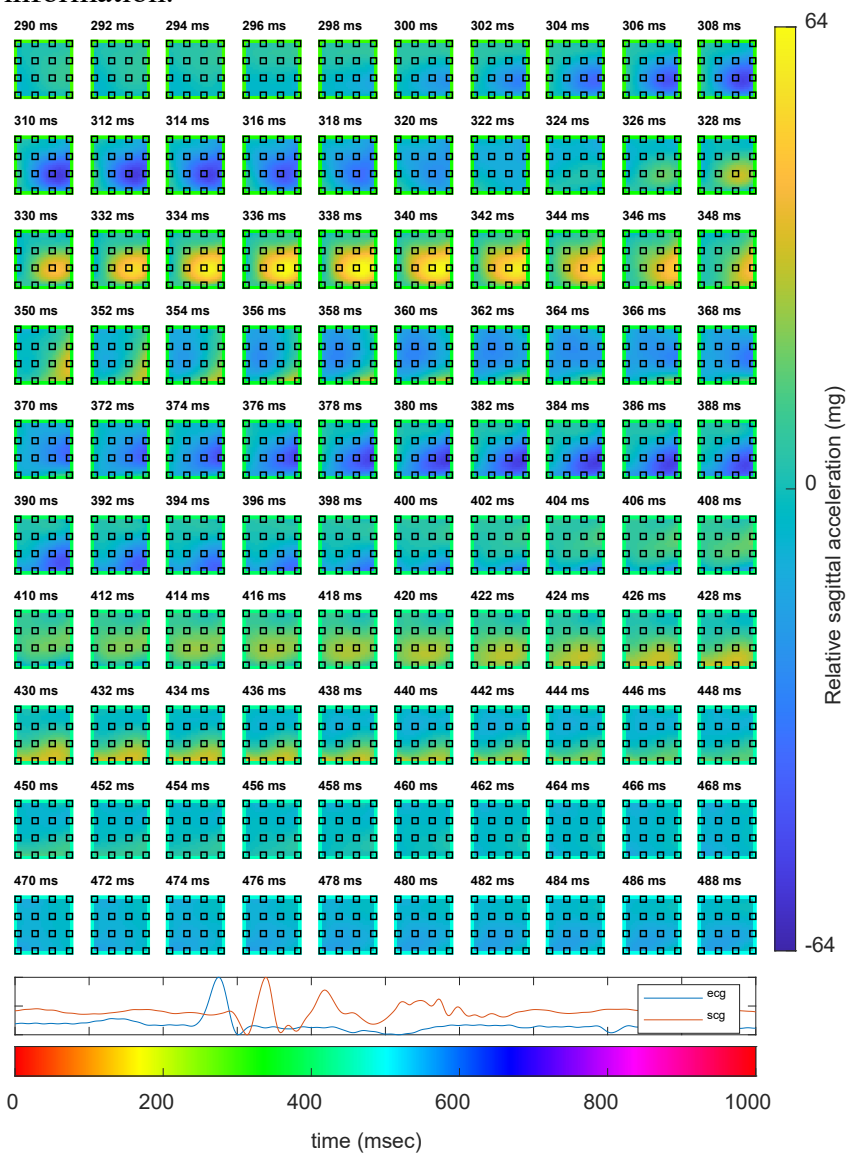

Figure 4 Snapshots examples of color plots for sagittal accelerations. Interval of snapshot are $2 \mathrm{~ms}$. Text above each plot and color around each color plots corresponds to a time relative to the colorbar and line plot. Squares represent the 16 accelerometers and spatial location. Data in figure is from subject 7 .

\subsection{Tracking map}

The tracking map is an expression of all dimension of the data. Thus, there is no need for addition figures to present all three-axis and time. However, due to the high compression of data, into one image, an investigator will need features to rotate and maneuver within its spatial dimensions. The maneuvering would help understand the multiple accelerometers collective vibrations.

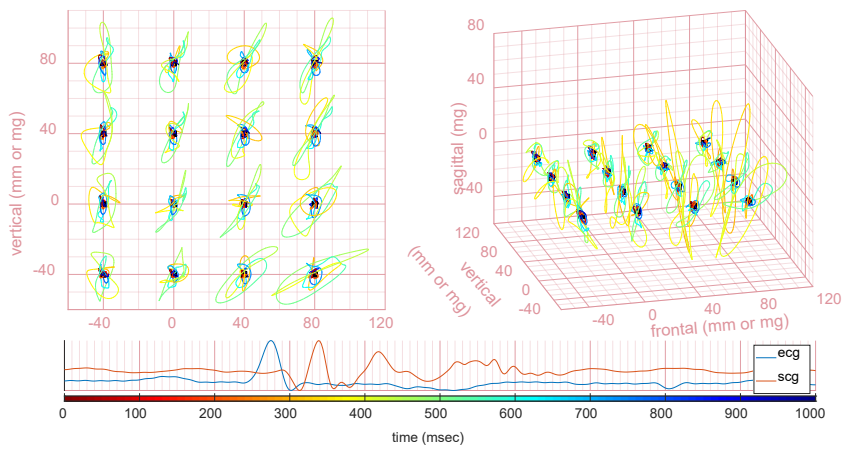

Figure 5 Example of acceleration tracking map for the frontal and vertical axes (left); and all axes (right). Black squares mark accelerometer locations. The frontal and vertical axes are in units of $\mathrm{mm}$ for accelerometer location and $\mathrm{mg}$ for acceleration. Each part of the trajectory is colored such that they correspond to a time relative to the colorbar and line plot. Data in figure is from subject 7 .

\subsection{Seismic mesh}

The seismic mesh shows the three cardinal axes collective vibration and gives a detailed information about the wavelengths of the vibrations by interpolation. However, since this method does not include time in the figure, it requires video or snapshots for presentation.

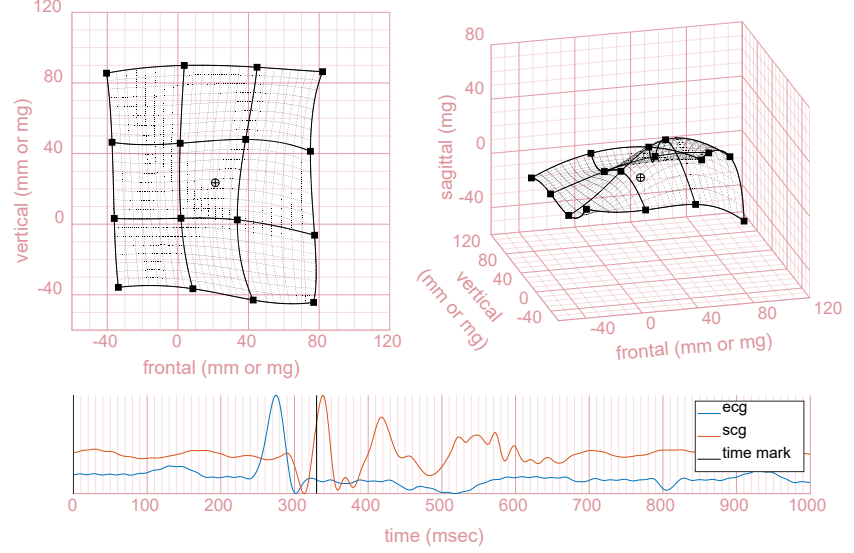

Figure 6 Example of acceleration seismic mesh for the frontal and vertical axes (left); and all axes (right). Black squares mark accelerometer locations. Current time frame is marked in the bottom plot. The frontal and vertical axes are in units of $\mathrm{mm}$ for accelerometer location and $m g$ for acceleration. Data in figure is from subject 7 . 


\section{Discussion}

We have implemented 4 visualization methods based on existing methods for similar biomedical modalities and vibration studies. Then we made a summarization of different methods that was able to map chest wall vibrations, and obtained chest wall vibration maps from 11 subjects with a mchSCG equipment. Finally, we investigated the visualizations methods strengths and weaknesses with the chest wall vibrations, characterized by the temporal and spatial capabilities.

For spatial characteristics the SCG chart has the lowest resolution as oppose to both the color plot and seismic mesh, that use interpolation to increase the resolution. The spatial interpolation helps with interpretations of the spatial behaviors of the vibration waves, because of the slowed development of vibration waves movement. Only the tracking map and seismic mesh includes information from all dimensions in one visual representation. The main differences between these methods are that the tracking map plots the acceleration in all three dimensions, while the seismic mesh includes this spatial information through the distance between the interpolation lines. However, the seismic mesh does show the combined three-axis information in a way that is as simple to interpret as a collective movement, compared to the tracking map.

Both the tracking map and the seismic mesh show a behavior where the accelerometers collective vibrations are following each other. This common motion is dominant in the vertical axes and becomes clearer in the seismic mesh were the mean collective vibration of all accelerometers are shown.

Temporal features are best observed with the SCG chart where temporal alignments between signals from the different measurement points can be studied. The tracking map and seismic mesh relies in either video or multiple snapshots for temporal illustration which are not well suited for papers, but ideal for computer added visualization where users have maneuvering options.

The seismic mesh is limited by the spatial resolution of the accelerometer formation. Since the accelerometers are 30 by $30 \mathrm{~mm}$, the shorter wavelength gets damped [11].

\section{Conclusion}

The purpose of the study was to investigate methods useful to visualize high dimensional data of physical event. SCG charts are good for temporal information, color plots are good for single axis spatial information, tracking map are great for directionality, and seismic plot is great for all axis spatial information. We believe that the visualizations can be useful for interpretation of the chest wall vibrations related to cardio-mechanic events.

\section{References}

[1] P. Mounsey, "Praicordial ballistocardiography," $\mathrm{Br}$ Hear. Journace, vol. 19, no. 2, 1956.

[2] W. Y. Lin et al., "Identification of location specific feature points in a cardiac cycle using a novel seismocardiogram spectrum system," IEEE J. Biomed. Heal. Informatics, vol. 22, no. 2, 2018.

[3] M. Cozig, L.-G. Durand, and R. Guardo, "Development of a cardiac acoustic mapping system," in Proceedings of 17th International Conference of the Engineering in Medicine and Biology Society, 1995.

[4] J. J. Struijk et al., "Heart-valve sounds obtained with a laser doppler vibrometer," in Computing in Cardiology, 2016, vol. 43

[5] P. Shirkovskiy et al., "Airborne ultrasound surface motion camera: Application to seismocardiography," Appl. Phys. Lett., vol. 112, no. 213702, 2018.

[6] F. Nogata, Y. Yokota, Y. Kawamura, H. Morita, and Y. Uno, "Visualization of heart motion by analysis of chest vibration," in IFMBE Proceedings, 2011.

[7] K. M. Jeppesen, "Development of a novel multichannel seismocardiography method," Aalborg University, 2017.

[8] K. Sørensen, S. E. Schmidt, A. S. Jensen, P. Søgaard, and J. J. Struijk, "Definition of fiducial points in the normal seismocardiogram," Sci. Rep., vol. 8, no. 1, 2018.

[9] S. E. Schmidt et al., "Three-dimensional apexseismocardiography," Comput. Cardiol. (2010)., vol. 41, 2014.

[10] Peter M. Shearer, "Surface waves and normal modes," in Introduction to Seismology, 2nd ed., Cambridge, 2009, pp. 215-256.

[11] E. B. L. Mackay, "Resource assessment for wave energy," in Comprehensive Renewable Energy, vol. 8, Elsevier Ltd., 2012, pp. 11-77.

Address for correspondence:

Kim Munck

Frederik Bajers Vej 7C

Aalborg, Denmark

kkj@hst.aau.dk 